This PDF is a selection from an out-of-print volume from the National Bureau of Economic Research

Volume Title: Evaluation of Econometric Models

Volume Author/Editor: Jan Kmenta and James B. Ramsey, eds.

Volume Publisher: Academic Press

Volume ISBN: 978-0-12-416550-2

Volume URL: http://www.nber.org/books/kmen80-1

Publication Date: 1980

Chapter Title: Some Comments on Papers by Dent and Geweke, Welsch, and Kelejian

Chapter Author: Saul H. Hymans

Chapter URL: http://www.nber.org/chapters/c11701

Chapter pages in book: (p. 219 - 222) 


\title{
Some Comments on Papers by Dent and Geweke, Welsch, and Kelejian
}

\author{
SAUL H. HYMANS \\ UNIVERSITY OF MICHIGAN \\ ANN ARBOR, MICHIGAN
}

The paper by Dent and Geweke continues the line of analysis of model specification which Christopher Sims (1972) developed on the basis of earlier work by Clive Granger (1969). The basic theorems--or implications of exogeneity, as they are referred to in this literature-are easily understood. If the vector $x$ is exogenous in a dynamic model determining $y$, then $y$ should be expressible as a true distributed lag in $x$, i.e., no leads in $x$ should matter. That, of course, is the "final form" for $y$. Further, whatever determines $x$, it cannot be $y$, so that an autoregressive equation in $x$ should permit no lagged $y$ s to be significant, the lagged $x \mathrm{~s}$ in effect eliminating any spurious correlation between $x$ and lagged $y$ s. These basic theorems are testable implications of the assumption of exogeneity of $x$. Dent and Geweke urge, as Sims has done for some time, that the process of building a time series econometric model should begin with a test of the exogeneity assumption. If the exogeneity assumption fails the test, it makes no sense to claim that the usual statistical properties hold for the procedures used to estimate the structural parameters of the time series model. The specific contributions of Dent and Geweke involve the determination of a joint test based on simultaneous consideration of the two implication theorems and the inclusion of a succession of identifiability and normalization tests for a model that passes the exogeneity test. The latter results appear not to be new, except in the sense of the suggestion that they be regarded as steps in a logical process of pretesting a hypothesized structure.

Before turning to the question of how important exogeneity testing is apt to be for the real world of model building, a few warnings about the use of the procedure of trying to falsify the implication theorems by finding 
significant leads of $x$ in the $y$-on- $x$ equation or significant lags of $y$ in the $x$-onlagged- $x$ equation may be listed:

(1) To be valid, the tests must include a greater number of theoretically extraneous leads on $x$ and lags on $y$ the greater the sample size. Inevitably, some leads on $x$ and some lags on $y$ will appear statistically significant with a probability approaching unity as the sample size increases. One has to resist the temptation to look for so-called significant coefficients. The key is to stick with the joint test that a number of vectors of coefficients differ significantly from null vectors.

(2) A second warning is that although the suggested procedure for testing exogeneity is robust with respect to the structural specification of the model, it is highly dependent on the assumption of linearity of the model and additivity of the disturbances. This is not unique to exogeneity testing.

(3) A third warning is that although the model builder should feel better about building the model after the $x$ s have passed the exogeneity test, parameter estimation on the same data set fails to satisfy classical specification axioms. In a sense a version of the preliminary test estimator problem would seem to exist.

Now let us turn to the question of the benefits that model builders and model users might derive from exogeneity testing. It can be argued that for microeconometric models the benefit is apt to be substantial in many cases. For macroeconometric models, on the other hand, I submit that exogeneity testing is almost without importance. Taking the latter case first, it can be claimed that macroeconometric modelers already know that the set of variables which are both partially correlated with the ys and exogenous is virtually a null set. If Dent and Geweke had not rejected exogeneity in the Haavelmo prewar and postwar models and in Klein's Model I, I would have suggested that something was wrong with their computer program. False attribution of exogeneity to a fast-shrinking and already-small set of variables is absolutely the least of the problems facing the Michigan Model or the Wharton Model or the DRI model. With respect to macro models, the Dent-Geweke conclusion that as exogeneity testing "becomes widespread, fewer but more durable simultaneous equation models ought to appear," is simply not to be taken seriously. We shall not have a small number of durable models until (a) we get much richer dynamic economic theory, (b) we find out a great deal more about how to test between alternative nonnested structures, (c) we determine the small sample properties of nonlinear estimators in nonlinear models, (d) we learn more about estimating simultaneous equation models using $y$ s as instruments rather than $x$ s, and so on. In other words, the creation of a small number of durable macro models is still a goal which we are far from achieving, and exogeneity-testing brings us no closer to it than we would be without it. 
Once this is said, it begins to become clear why exogeneity testing may well be important in microeconometric modeling. Disposable income has a very good chance of being quantitatively, hence statistically, exogenous to a model of the onion market. And it is probably exogenous to a model of the meat market, and it is sensible to hypothesize and then test whether or not it is exogenous to a model of the furniture market. Obviously, nonlinearity problems exist in micro models as well as in macro models. But there is a chance that the micro nonlinearities can be localized in the candidate exogenous variables. And if disposable income is exogenous, so is its square or square root or logarithm. So I see a real and vital role for this area of analysis and I am glad theoretical results are being developed. Unfortunately, I do not see it having any impact in that branch of model building which absorbs most of my time and happens to need a great deal of careful evaluative analysis.

This leads me quite naturally to the work on robustness and various aspects of data anlaysis that is being carried on by a number of researchers and exemplified in the paper of Roy Welsch. The approach here is not limited to time series models or to macroeconometric models, but I should like to comment on Welsch's work in that context because-as I indicated earlierthe need for careful evaluation of macroeconometric models is very substantial. Welsch is right to feel that those of us who build such models know too little about our data. It is hard for us to get the same feel for 75 observations on 6 or 8 variables multiplied by a three-digit number of equations as could be achieved in the good old days of working with a couple of scatter diagrams. In addition, a two-year distributed-lag was $x_{t}$ and $x_{t-1}$ in the old days of annual models, and it is $x_{t}, x_{t-1}, \ldots, x_{t-7}$ in current quarterly models. In another sense, those of us who build macro models also know too much about our data. It is not hard to find a configuration of variables and/or a pattern of lags which will get rid of what we know is a troublesome observation in a simpler specification.

Suppose one starts with the presumption that the model being built involves serious misspecifications and too many dimensions for profitable use of elementary descriptive statistics. The presumption of misspecification forces one away from evaluative recipes which are too heavily tied to classical parametric methodologies and leads one to search out nonparametric tests of model reliability - primarily descriptive kinds of procedures: root mean squared errors, Theil's $U$-statistic, regressions of actual on predicted values, turning-point errors, whatever. Welsch's work makes what may be a very valuable addition to this tool kit of descriptive procedures. He develops a battery of diagnostic descriptors which can be applied to equations that are likely to involve misspecification and that are estimated over a large number of variables. It is unlikely that all the descriptors will yield to sophisticated, independent parametric testing, but they boil a big multidimensional problem 
down to manageable, often visually manageable, proportions. And the descriptors - which are heavily interrelated - seem likely, in combination, to reveal a good deal about the sensitivity of the statistical outputs to various input perturbations. Obviously much more needs to be done since as yet the descriptive diagnostics are not terribly prescriptive. But I am impressed that Welsch and his co-workers are pursuing a useful line of attack, and I believe that procedures of this kind will get a pretty good workout from quite a number of us for a long while before they are all obviated by any major theoretical breakthroughs enabling us to handle model validation in a systematic parametric form.

Finally, let me offer just a few observations on the paper by Kelejian. As usual in papers dealing with aggregation, one comes away depressed about how nasty the problem is. But if the paper depressed me, it also impressed me. Kelejian's approach seems to have drawn profitably from the otherwise rather unproductive literature on aggregate production functions. The aggregation results that Kelejian derived in the limit as the number of micro units increases put me in mind of the earlier paper by Ramsey (1972), cited by Kelejian. In his paper Ramsey derives an approximation to the market demand curve which follows from $N$ micro stochastic demand curves as $N \rightarrow \infty$. Ramsey and Kelejian came at the problem really quire differently but derived very similar limiting aggregates. Compared with Kelejian, Ramsey is more general in requiring only independence, but not identical distribution, across the micro functions, and Ramsey's result is not dependent on a specific functional form for the micro functions. Kelejian, on the other hand, is more general in permitting stochastic independent variables in the micro functions. Yet Kelejian's Eq. (12) - the exact limiting macro function corresponding to the precisely specified micro set in Eq. (4)-is, for all practical purposes, Ramsey's Eq. (25) - the approximation to the limiting macro function derived without exact prior specification of the micro functions.

Perhaps Kelejian and Ramsey will get together and figure out what is happening. Maybe in the aggregate they can write the happy aggregation paper we are all waiting for.

\section{REFERENCES}

Granger, C. Investigating causal relations by econometric models and cross-spectral methods, Econometrica 1969, 37, 428-438.

Ramsey, J. Limiting functional forms for market demand curves, Econometrica 1972, 40, $327-342$.

Sims, C. Money, income and causality, American Economic Review, 1972, 62, 540-552. 